\title{
A Physiological-Monitoring Electronic Platform for Cattle Grazing Systems
}

\author{
Ricardo R. Santos, UFMS, Campo Grande, Brazil \\ Fabiana V. Alves, EMBRAPA, Campo Grande, Brazil \\ Patrik O. Bressan, IFMS, Campo Grande, Brazil \\ (iD) https://orcid.org/0000-0002-4903-2674 \\ Ricardo E. Aguiar, INDEXT, Campo Grande, Brazil \\ Wellington O. Santos, INDEXT, Campo Grande, Brazil \\ Rafael A. Costa, INDEXT, Campo Grande, Brazil
}

\begin{abstract}
In this work, we present a non-invasive electronic platform for physiological data acquisition on cattle grazing systems. The platform can be used for dairy and beef cattle to continuously monitor physiological variables such as skin temperature, heartbeats, and respiratory frequency. The set of sensors are coupled into a halter so that they are in touch with the animal's forehead. Users can monitor the data acquired by the electronic device using a mobile device (smartphone or tablet) and it visualizes important physiological parameters in the platform cloud system.
\end{abstract}

\section{KEYWORDS}

Cattle Welfare, Hardware Device, Physiological Monitoring, PPG Signals, Thermal Stress

\section{INTRODUCTION}

The current food consumption and production scenarios based on livestock indicate high demand for new technologies. The U.S. per capita beef consumption was 57 pounds in 2017, and 59 pounds was estimated in 2018 (Widmar, 2018). Brazil, one of the most important beef cattle producers, has improved its production in 10\% from 2016 (1,400,485 tons) to 2017 (1,534,397 tons) (Brazilian Beef Exporters Association, 2018).

In the livestock industry, animal physiology and behaviour monitoring are fields where technology can improve previous analysis and diagnosis of animal health state and welfare. As a consequence of increasing production, the demands for animal monitoring on health conditions and behaviour monitoring are also increasing. The USDA reports inform that in 2015, due to factors such as bovine respiratory, digestives, and other illnesses, the industry also suffered a tantamount loss of more than 3.8 million cattle (98\% of the total of cattle losses). The estimated cost of death loss was $\$ 3.87$ billion (USDA, 2015). 
One commonly adopted procedure to monitor physiological conditions on cattle is the visual method. This method uses experts who are able to recognize, while cattle grazing, if the animal is in thermal stress based on the respiration rate and behavior. Despite being a well-known and simple procedure, the visual approach lacks scale and accuracy, since it is a time-consuming, and error-prone method, requiring an expert to be on site monitoring the animal and taking notes of its activities.

Considering cattle grazing systems, there is a technological gap on fast, non-invasive, and continuous devices and technologies to monitor and evaluate livestock welfare and health. Automated monitoring of physiological and behavioural responses of animals has the advantages of reducing labor, increasing the frequency of observation, and reducing bias and observer influence, but automation requires specialized instrumentation (Eigenberg, Brown-Brandl, \& Nienaber, 2008).

In this work, we present a non-invasive electronic platform able to monitor physiological parameters on cattle grazing systems. The platform can be used for dairy and beef cattle to continuously monitor variables such as skin temperature, heart-beats, and respiratory frequency. The platform is comprised of an electronic device, a user mobile app and a web cloud system. The device is based on a set of non-invasive sensors and probes, coupled to the animal's halter. All sensors are connected to a small electronic processing and communication unit responsible for gathering all data, processing, and storing them locally. Users visualize all data using a mobile device (smartphone or tablet) or a web system. A detailed analysis on each animal or the herd can be done using the web cloud system.

To the best of our knowledge, this is a first approach towards a non-invasive and autonomous platform able to the online monitoring of physiological data on beef grazing cattle. The platform represents an advance of the state-of-the-art proposals since it presents a solution for long-term monitoring the cattle using a non-invasive device.

\section{RELATED WORK}

The increasing demand for automatize monitoring processes and to provide automatic information in livestock activities has motivated several proposals of technological solutions. There are commercial products such as the Polar Sport Tester, the Polar Equine RS800cx G3, focused on horses to monitor heart beat (Janzekovic, Prisenk, Mursec, Vindis, Stajnko, \& Cus, 2010), and devices to monitor cattle location and temperature (Caja, Castro-Costa \& Knight, 2016), among others. Livestock managers need information about how and why their animals respond to environmental challenges to make improved decisions on strategies to reduce animal production losses at hot weather. In order to minimize loss, many physiological stress indices are used, such as heart or respiration rate.

Silve et al. (Silva, Arce, Souto, \& Costa, 2005) present a wireless sensor network prototype to monitor physiological responses of livestock. They have measured the bovine brain electrical activity by capturing EEG signals. The equipment comprises two modules, one placed on the animal head (animal module) and the other in an observation station (base module).

Recent work (Sousa, Canata, Leme, \& Martello, 2016; Sousa, Rodrigues, Abreu, Tabile \& Martello, 2018) have applied machine learning methods to developed fuzzy and artificial neural network (ANN) models to predict thermal stress in beef cattle. Both classifiers (fuzzy and ANN) integrate weather and animal factors to assess individually the level of thermal stress in feedlot finishing cattle.

Noninvasive sensors have been a subject for development and research to collect data of heartbeat and Respiration Rate (RR). The manual method for RR counting in cattle (count the flank movement visually) is time-consuming, labor-intensive and subject for misinterpretations for a continuous measurement. Strutzke et al. (Strutzke, Fiske, Hoffmann, Ammon, Heuwieser, \& Amon, 2019) adopted a differential pressure sensor which is placed in a halter and positioned on the animal's jaw, fixed on the right side of the head. They use a flexible tube to capture the nasal exhalation pressure and to send to the pressure sensor. The sensor has been tested and evaluated under controlled conditions 
and for a small number of dairy cattle, but it seems difficult to apply such proposal to large herds and even for grazing cattle.

The large technology usage and applications are shown in the review presented in (Talavera, Tobón, Gómez, Culman, Aranda, Parra, Quiroz, Hoyos \& Garreta, 2017). The authors point out that some of the challenges and gaps for Internet of Things (IoT) systems applied to livestock are battery life span, interoperability, and costs. One can note that there is a gap on IoT platforms focused on cattle physiological signals sensing and monitoring. Their observations show that the adoption of monitoring technology in cattle farming is still a challenge. The technological gap is even larger if we consider the automatic acquisition and analysis of physiological signals such as beats-per-minute (BPMs), $\mathrm{RR}$, temperatures, and the correlations to environmental data to predict welfare and health state.

Another survey is presented in (Sharma \& Koundal, 2018) where they identify eleven systems for cattle health monitoring. Despite the number of proposed solutions, they informed that there are still a lot of challenges faced by farmers such as adopting technologies for early and accurate detection of diseases and long-term monitoring.

\section{ELECTRONIC PLATFORM}

We have designed an electronic platform comprised of a device that is used by the animal, a mobile app to capture physiological data and a web cloud system. The device has a set of sensors (oximeter and skin temperature termomether), electronic board, wireless communication and an autonomous energy system allowing a user to monitor and analyze physiological data acquired from an animal in real time. All the sensors are non-invasive so that the system interference on the animal's behaviour and health conditions is inconspicuous.

All acquired data are stored into the electronic board where a user has access using a smartphone or tablet. The user software is able to setup start and end times to acquire data. The data in the mobile software can be also synchronized to a web platform (homolog version available at https://bep-homolog.firebaseapp.com/) to allow the user to analyze the data from an individual or a group (Figure 1).

Figure 1. The electronic platform usage. Electronic circuits and sensors are into a case placed on the front head band.

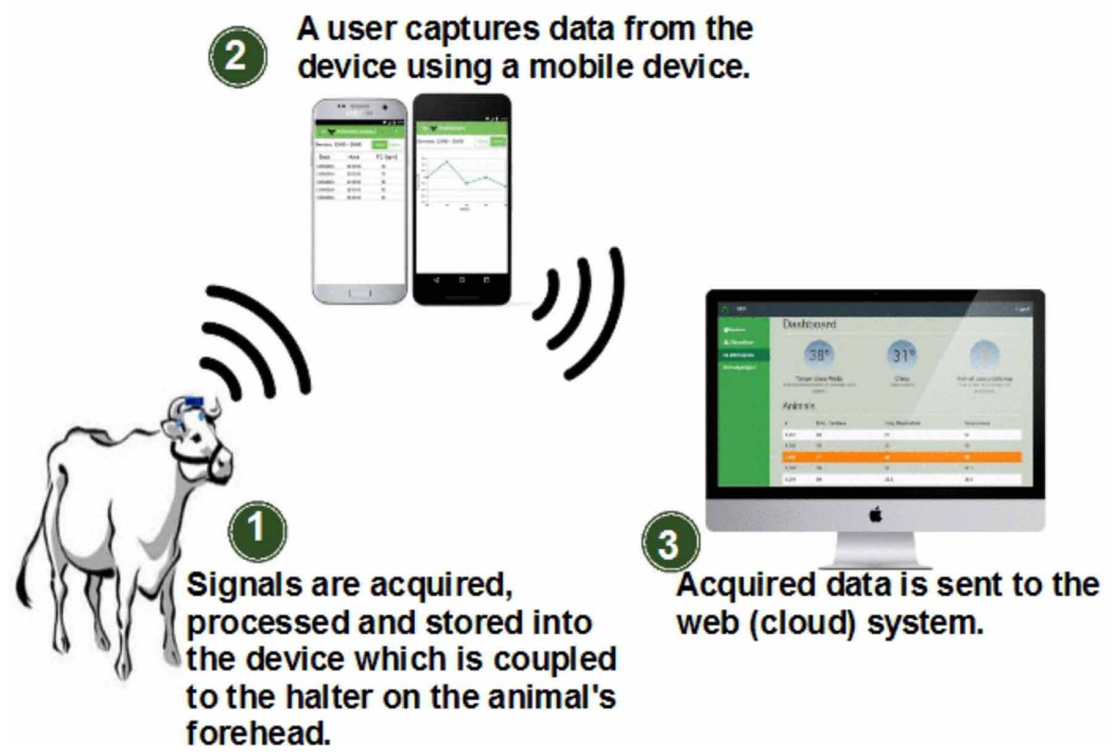


The challenges involved with a platform focused on get useful information from physiological data are mostly related to the design of accurate algorithms working on raw data. In this work we have designed algorithms to get Beats-Per-Minute (BPM) and Respiration Rate information from cattle PPG signals.

\section{BEATS PER MINUTE ALGORITHM}

We have adopted a non-invasive oximeter sensor to capture blood flows signals - the photoplethismography (PPG) signals - and extract out the Beats Per Minute (BPMs) and Respiration Rate (RR). The PPG waveform is presented in Figure 2. Each PPG cycle has a start point T, a peak $\mathrm{P}$, and the waveform goes down to point $\mathrm{D}$ (dicrotic notch).

Figure 2. PPG waveform interests points: initial point (T), peak (P), and dicrotic notch (D)

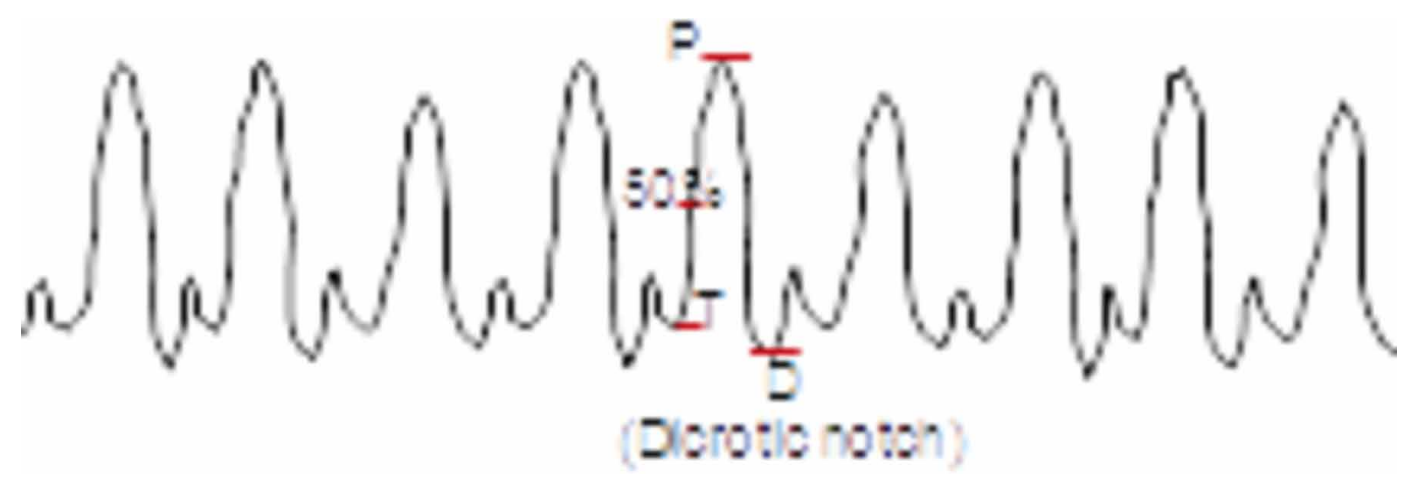

The BPM algorithm finds out the start of the PPG cycle and calculates the time between each PPG cycle (Inter Beat Interval - IBI). IBI means the blood flow pumped from the heart, using arteries, to all body extremities. By looking at the interest points $\mathrm{T}$ and $\mathrm{P}$ in a waveform, an algorithm can identify each PPG flow along the time and extracts out the BPM in each time interval (Reisner, McCombie \& Asada, 2008). Our BPM algorithm is able to calculate the IBI from a cattle PPG waveform. From our experiments on cattle PPG signals, we have adopted $50 \%$ of the PPG cycle (Figure 2) to start counting a cattle heartbeat. Algorithm 1 presents the steps to obtain BPM from the PPG waveform.

Algorithm 1: BPM ALGORITHM

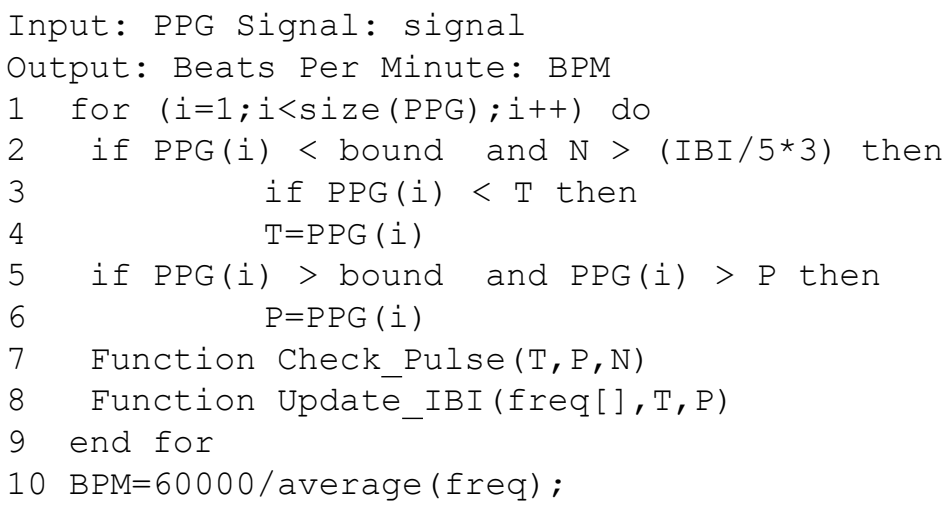


Algorithm 1 calculates the BPM by reading all the PPG data in the PPG array (rows 1-9). Object $\mathrm{N}$ is a time counter (microseconds) representing the time of the heartbeat cycle. Objects $\mathrm{P}$ and $\mathrm{T}$ represent the max and min values for the current PPG cycle (Figure 2). Given that a PPG waveform uses all our ADC (Analog-Digital Converter) representation range, Bound is initialized with half $(50 \%)$ of the range value of the ADC circuit. After $60 \%$ (3/5) of the IBI, T is updated to avoid noises and false positive reads of the dicrotic notch. Function Check_Pulse evaluates N, P and T to identify if there is a valid heartbeat pulse. Function Update_IBI updates array frequency with the last IBI from points $\mathrm{P}$ and $\mathrm{T}$. Row 10 calculates the BPM from the IBIs in the frequency array.

One can observe that some parameters such as N, bound, and IBI are very specific to our application in cattle. From our experiments, the range of PPG values are a bit different (larger) than in humans so that is necessary to adopt an ADC circuit that represents all the PPG data acquired from the animal thus forcing a calibration of some parameters in Algorithm 1.

\section{RESPIRATION FREQUENCY ALGORITHM}

The respiration cycle can also be extracted out from the PPG cycle (Meredith, Clifton, Charlton, Brooks, Pugh \& Tarassenko, 2011). Based on the relationship between the PPG waveform and the respiration, an algorithm proposed in (Lazaro, Gil, Bailon, \& Laguna, 2011) calculates the respiration rate from the PPG amplitude and width variability. At expiration time, the parasympathetic system makes the blood vessels more flexible than the inspiration. The blood vessels flexibility reduces the waveform propagation velocity also affecting the PPG waveform width. Conversely, at inspiration time, the sympathetic system increases the arterial stiffness, thus increasing the waveform propagation velocity. This behaviour shows us that the PPG waveform width depends on the respiration so that the Pulse Width Variability (PWV) can be used to indicate respiration information on the individual. Figure 3 presents the interest points $(\mathrm{Na}, \mathrm{Ne}, \mathrm{No})$ for an RR calculation in a PPG waveform: $N_{A i}$ is the waveform peak (apex) in pulse $i ; N_{O i}$ is the waveform start (onset) in pulse $i ; N_{E i}$ is the waveform end (endset) in pulse $i$.

Figure 3. Respiration rate (RR) points of interests in a PPG waveform

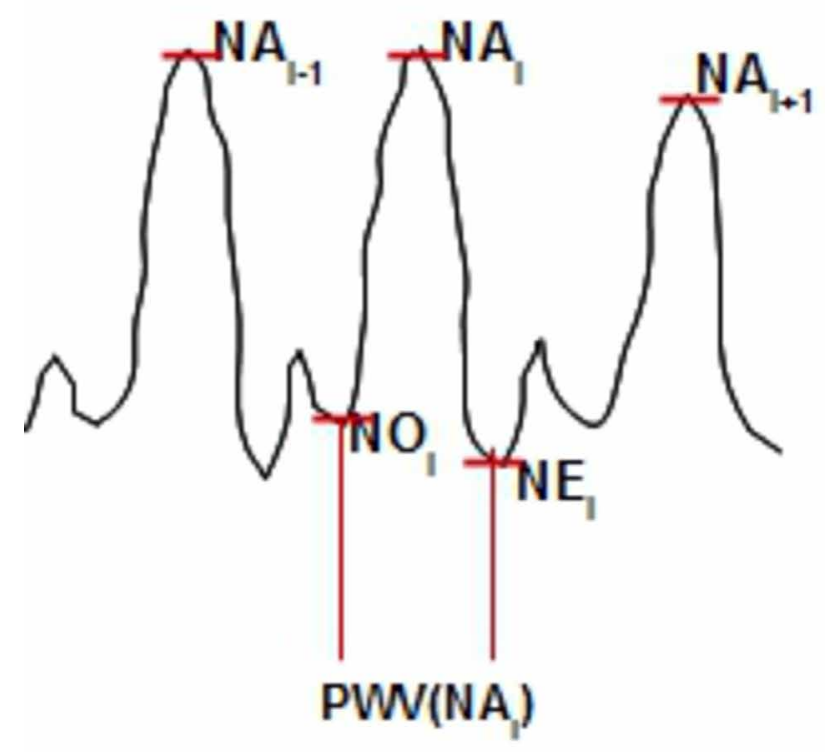


We have developed the PWV algorithm to extract out RR from the original PPG waveform in cattle grazing systems. Our algorithm looks for the interest points (Figure 3) in the PPG waveform to obtain a new PWV waveform. The RR information (RR cycles) can be easily obtained by reading the cycles of the PWV waveform. Algorithm 2 gets a PPG waveform as input and provides three arrays $\mathrm{Na}$, No, and Ne representing the interests points in Figure 3.

Algorithm 3 is based on the work of Lazaro et al. (Lazaro, Gil, Bailon, \& Laguna, 2011; Lazaro, Gil, Bailon, Minchol \& Laguna, 2013) and it obtains the PWV waveform from the interests points (Algorithm 2). Once a PWV data is available, the RR is obtained by calculating the cycles. The cycle heights are the variability of the original PPG waveform so that they represent the respiratory cycles. The RF information can be easily obtained by reading those respiration cycles and the time intervals of each cycle.

\section{Algorithm 2: POINTS OF INTERESTS IDENTIFICATION ALGORITHM}

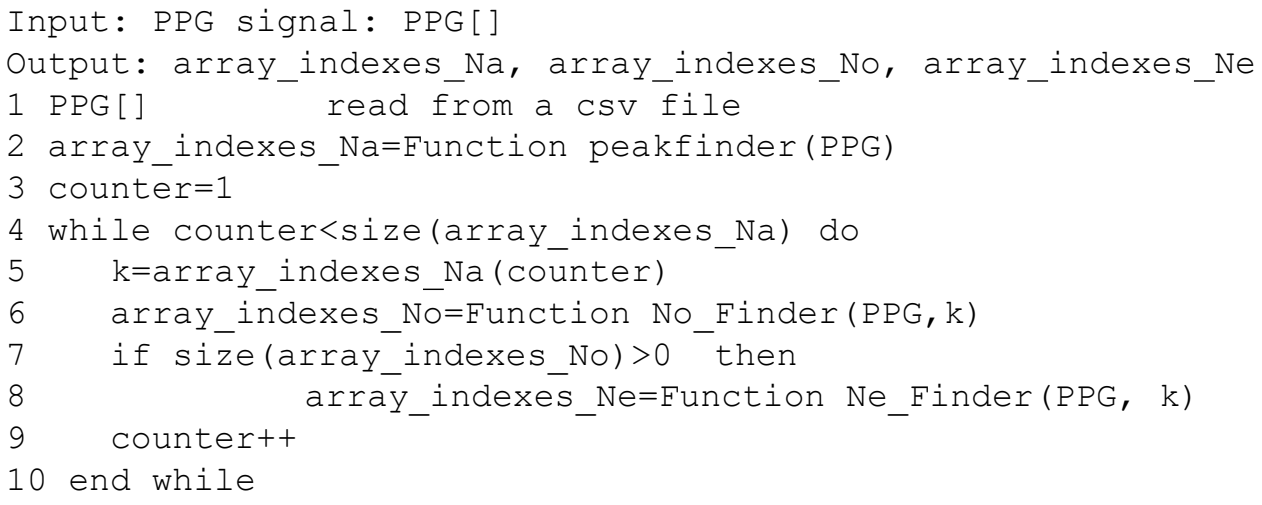

In Algorithm 2, the PPG waveform data is read from a CSV file (Comma Separated Values). Function peakfinder is the responsible for finding the peaks (signals and values representing the $\mathrm{Na}$ points) of the PPG waveform. From the Na indexes in the PPG waveform, the algorithm looks for No and Ne interest points (rows 4-10). Functions No_Finder and Ne_Finder return the array of No and Ne points found in the PPG waveform taking the Na point $(\mathrm{k})$ as the baseline. Considering that the waveform could be no fitted to the algorithm, there is a check if the algorithm found a No point (row 7) so that it seems to be able to find a Ne point.

\section{Algorithm 3: PWV WAVEFORM FROM THE INTERESTS POINTS}

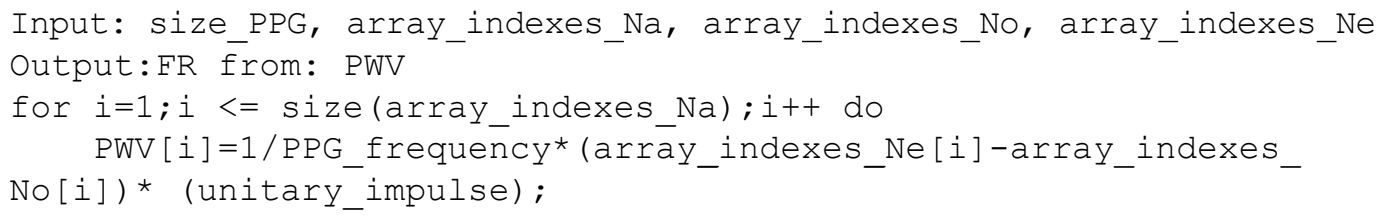

Algorithm 3 runs on arrays $\mathrm{Na}, \mathrm{Ne}$, and $\mathrm{No}$ (the three arrays have the same size) in order compose the PWV waveform from width of the Ne and No points, the cycle time (1/PPG_frequency) used to acquire the PPG data and the unitary impulse. The unitary impulse has been implemented according to (Lazaro, Gil, Bailon, Minchol \& Laguna, 2013). The PWV constitutes a new waveform using the time of the PPG width. The peaks of this new waveform form up cycles which are the variability of the PPG width thus representing the respiration cycles. 
Since the PPG data is acquired from a moving grazing animal, there are unavoidable noises in the original PPG waveform. Those noises disturb the PPG waveform and are mostly identified when there are clipped off signals or sequences of zero signals. Our algorithm filters out those disturbing signals before calculating BPM and RR. Even so, for unwanted signals that can disturb the PPG waveform, our algorithm of points of interest checks out arrays No and $\mathrm{Ne}$ in order to identify if valid signals were identified.

\section{DEVICE PROTOTYPE}

Figure 4 shows animals using the device while they are grazing. All electronic circuits and sensors are placed into a plastic case, and the case is on a forehead band, fixed to the halter. Figure 5 sketches the electronic system and the acquisition data flow.

Figure 4. Animals grazing and using the electronic platform on the forehead
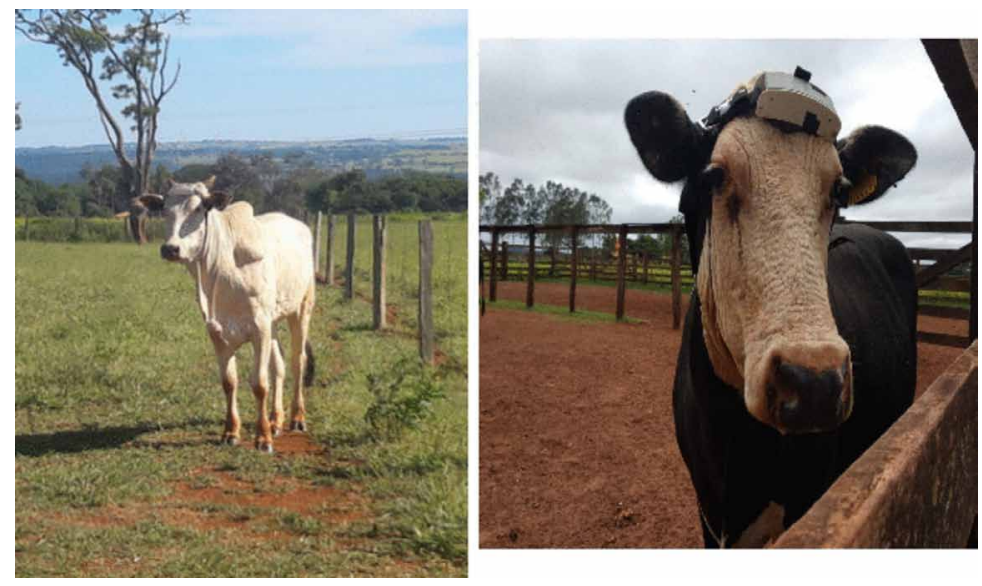

Components 1) and 2) are the physiological sensors (temperature and oximeter), battery, and the electronic circuits to acquire, process, and store data. The sensors are a reflectance oximeter and a skin temperature sensor. From our observations on the animal's data and the usage of oximeter on this application, we have designed in house circuits for signal conditioning able to acquire, filter, and convert the analog data to digital data. The skin temperature sensor is packaged into an ibutton model DS1921G since it offers compliance with medical standards. We have designed a software and hardware interface so that the ibutton is fully controlled (programming and collecting data) by our platform. In experiments using data acquisition at $5 \mathrm{~min}$, the device is able to store data longer than 2 weeks and the battery needs a recharge after 1 month of usage. The processing, synchronization, and control in the device is performed by an ATMEGA 1284P System-on-Chip (SoC) circuit.

Component 3) (User mobile device) is responsible for acquiring data from the electronic device. The algorithms for Beat-Per-Minute and Respiration Rate run in the user mobile device. The communication between the mobile and the animal's device is performed by Bluetooth so that there is no need for wifi signal or even high cost network infrastructure in the farm. Once the communication is started, the data is encoded transmitted and locally stored into the mobile device. The mobile application calculates the BPM and RR physiological data for each animal and it highlights alert information when those data are out of the normal range. From the mobile application, the user is able to turn on/off the electronic device and send the physiological data to the cloud system. 
Figure 5. Electronic platform block diagram

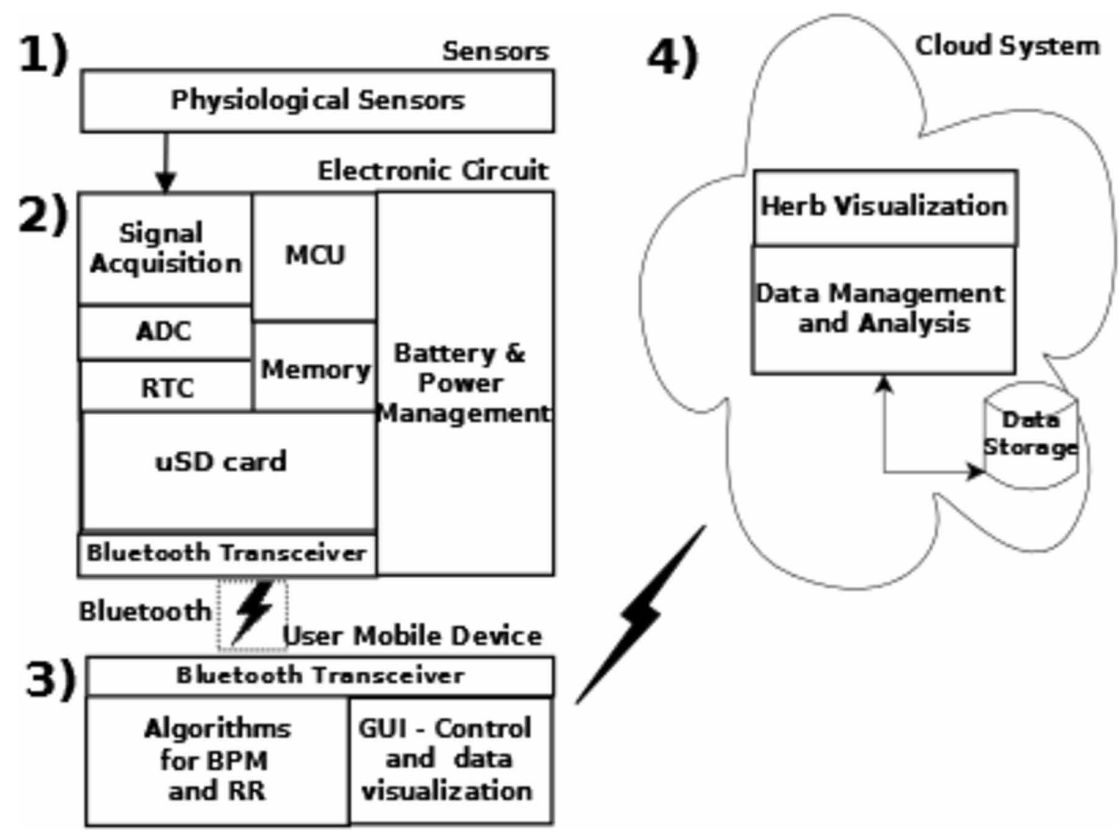

The fourth component is a web platform that provides data analysis, individual, and herd data visualization. The mobile device sends the data as a JSON format file to a Firebase database in the cloud system. The web application gets the data from the Firebase and it performs physiological graphical analyzes considering each animal and the herd according to the previous registers that the user has done in the system.

Figure 6 presents the device circuit boards (1) and the case with all the circuits and battery (4). The printed circuit boards are responsible for connecting all sensors to the processing, storing and

Figure 6. Front side of the electronic circuit boards and case used in the device
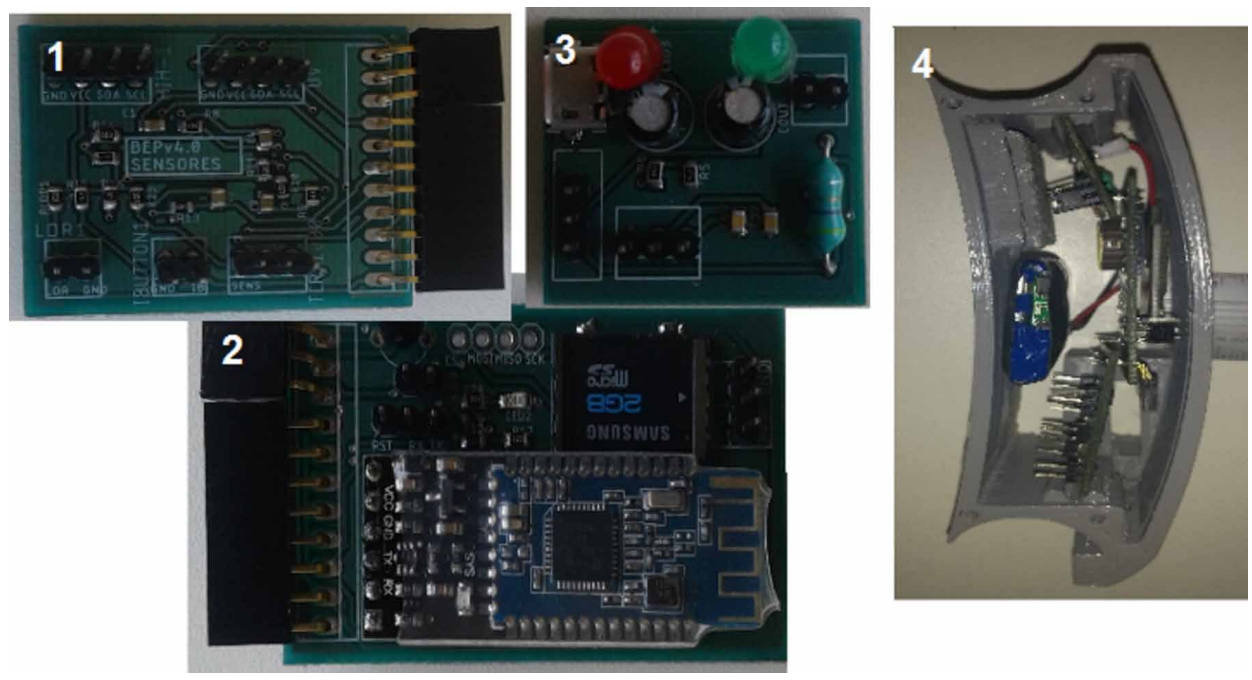
communication circuit (2), and managing the battery power supply (3). The sensors are exposed out of the case so that they are in touch to the animal's forehead.

\section{EXPERIMENTS AND RESULTS}

The experiments using our electronic platform have been performed on four animals from the Nelore zebu breed. The animals were grazing while the platform was acquiring the data. Our goal was to estimate BPM and RR from each animal using our platform and comparing the results to a visual (manual) observation of those parameters. The manual acquisition data was performed by a veterinarian using stethoscope (in intervals of $5 \mathrm{~min}$ ) and manual respiration counting. The animals were using the device for the first time and they had an active behaviour so that we have decided to capture data for a short period (about $1 \mathrm{~h}$ ). Despite our efforts to design a case and halter able to accommodate all electronic circuits and sensors and be comfortable to the animals, we have observed that signal noises in the PPG signal come up mostly from the lack of a fixed positioning of the oximeter sensor. As the sensor moves according to the animal's movement, some spurious signals disturb BPM and RR algorithms estimates.

Table 1 presents the data from the four animals in the experiment. The acquired PPG signal has been used to calculate BPM and RR information (Automatic Acquisition column). The Manual Acquisition column shows BPMs and RRs manually acquired from an expert. The BPM and RR values presented in Table 1 are averages considering the number of valid PPG samples (last column) acquired in the period and they were validated using a confidence interval of $95 \%$. The temperature values are also averages acquired in intervals of 1 minute along the period. For comparison purposes, we calculated the average error (\%) from the automatic acquisition as follows:

Error=(Manual_acquisition_value - Automatic_acquisition_value)/ Manual_acquisition_value

One can note that there are differences in BPMs between the manual and automatic acquisitions. Such differences are mostly due to the noises in the short time interval where the PPG signals were

Table 1. BPM and RR data from four animals

\begin{tabular}{|c|c|c|c|c|c|c|c|c|c|c|}
\hline & \multirow[t]{2}{*}{ Gender } & \multirow[t]{2}{*}{ Age } & \multicolumn{2}{|c|}{$\begin{array}{c}\text { Manual } \\
\text { Acquisition }\end{array}$} & \multicolumn{3}{|c|}{ Automatic Acquisition } & \multicolumn{2}{|c|}{$\begin{array}{c}\text { Average } \\
\text { Error (\%) }\end{array}$} & \multirow{2}{*}{$\begin{array}{c}\text { Number } \\
\text { of } \\
\text { Samples }\end{array}$} \\
\hline & & & BPM & $\mathbf{R R}$ & $\operatorname{Temp}(\mathrm{C})$ & ВPM & $\mathbf{R R}$ & BPM & $\mathbf{R R}$ & \\
\hline Animal 1 & female & 3 years & 40 & 25 & 38 & 36 & 28 & 10 & 12 & 50140 \\
\hline Animal 2 & female & 1 month & 36 & 28 & 37 & 41 & 31 & 14 & 11 & 58790 \\
\hline Animal 3 & female & 4 years & 32 & 29 & 39 & 36 & 32 & 12 & 10 & 48860 \\
\hline Animal 4 & male & 3 months & 32 & 24 & 39 & 34 & 25 & 6 & 4 & 176490 \\
\hline
\end{tabular}

acquired. When the experiment has more samples (row Animal 4), our algorithms work better and the average error is more accurate compared to the manual acquisition. We believe that on longer experiments, the animal may feel more comfortable using the device so that the PPG signal will have less noises.

Despite the differences in BPMs and RRs results, the experiment shows that the electronic platform is a useful tool to monitor cattle physiological parameters in a non-invasive way. Such device has 
shown as a viable, automated, and low-cost alternative to manual and time-consuming procedures to monitor and evaluate animal's welfare.

\section{CONCLUSION AND FUTURE WORK}

This work presented an electronic platform for physiological signals monitoring on cattle. The platform provides information concerning beats per minute, respiratory frequency, and skin temperature. Such information is essential to farmers who want real-time and non-invasive technological solutions for monitoring herds health state.

Besides the electronic circuit and sensors, we have also developed algorithms that determine BPMs and estimate the respiratory frequencies from PPG signals. Our algorithms have been tested and compared to manual measurements on four animals. The experiments showed an average error ranging from $4 \%-14 \%$ comparing the manual acquisition and our approach. We believe that at longer experiments where the animals can feel more comfortable using the device, those average errors will be lower. The experiments also showed that the non-invasive approach, based on an oximeter sensor coupled to the animal's halter, provides rather useful information to evaluate the animal health while the animal is freely grazing.

The electronic device can be used by applications focusing on automatically identifying thermal stress and welfare conditions from the BPM and RR data. It can be also used as a support tool to aid decision-makers on providing more accurate information, allowing for precise animal monitoring, that can optimize diets and other management practices that lead to improved animal welfare and performance.

One of the challenges towards a large-scale adoption of such technology to continuously perform the animal's monitoring is to improve the coupling between circuits and sensors thus keeping animal's comfort. As future work, we will focus on improving sensors precision, algorithms filtering and prediction accuracy, and correlating the data with animal's welfare and stress indexes.

\section{ACKNOWLEDGMENT}

The authors thank Brazilian Research Agencies FUNDECT, CAPES, and CNPq, for their financial support to this work. They also thank their institutions Federal University of Mato Grosso do Sul (UFMS) and Brazilian Agricultural Research Corporation/Embrapa Beef Cattle for their infrastructure and logistics support to this work. 


\section{REFERENCES}

Brazilian Beef Exporters Association. (2018). Brazilian beef exports. Retrieved from http://abiec.siteoficial.ws/ download/estatisticas-270218.pdf

Caja, G., Castro-Costa, A., \& Knight, C. H. (2016). Engineering to Support Wellbeing of Dairy Animals. The Journal of Dairy Research, 83(2), 136-147. doi:10.1017/S0022029916000261 PMID:27210489

Eigenberg, R., Brown-Brandl, T., \& Nienaber, J. (2008). Sensors for Dynamic Physiological Measurements. Computers and Electronics in Agriculture, 62(1), 41-47. doi:10.1016/j.compag.2007.08.011

Florentin, D. (2010). Estimación y Evaluación de la Señal Respiratoria a partir de la Señal Fotopletismográfica de Pulso, Señal de Presión Arterial y Señal Electrocardiográfica [Master dissertation]. Universidad de Zaragoza. Centro Politécnico Superior.

Janzekovic, M., Prisenk, J., Mursec, B., Vindis, P., Stajnko, D., \& Cus, F. (2010). The Art Equipment for Measuring the Horse's Heart Rate. Journal of Achievements in Materials and Manufacturing Engineering, 41(1-2), 180-186.

Lazaro, J., Gil, E., Bailon, R., \& Laguna, P. (2011). Deriving Respiration from the Pulse Photoplethysmographic Signal. Computers in Cardiology, 713-716.

Lazaro, J., Gil, E., Bailon, R., Minchol, A., \& Laguna, P. (2013). Deriving Respiration from Photoplethysmographic Pulse Width. Medical \& Biological Engineering \& Computing, 51(1-2), 233-242. doi:10.1007/s11517-0120954-0 PMID:22996834

Meredith, D. J., Clifton, D., Charlton, P., Brooks, J., Pugh, C. W., \& Tarassenko, L. (2011). Photoplethysmographic Derivation of Respiratory Rate: A Review of Relevant Physiology. Journal of Medical Engineering \& Technology, 1-7. PMID:22185462

Reisner, A., Shaltis, P.A., McCombie, D., \& Asada, H.H. (2008). Utility of the Photoplethysmogram in Circulatory Monitoring. American Society of Anesthesiology, (5), 950-958.

Sharma, B., \& Koundal, D. (2018). Cattle Health Monitoring System Using Wireless Sensor Network: A Survey from Innovation Perspective. IET Wireless Sensor Systems, 8(4), 143-151. doi:10.1049/iet-wss.2017.0060

Silva, A. C. S., Arce, A. I. C., Souto, S., \& Costa, E. J. X. (2005). A Wireless Floating Base Sensor Network for Physiological Responses of Livestock. Computers and Electronics in Agriculture, 49(2), 246-254. doi:10.1016/j. compag.2005.05.004

Sousa, R. V., Canata, T. F., Leme, P. R., \& Martello, L. S. (2016). Development and Evaluation of a Fuzzy Logic Classifier for Assessing Beef Cattle Thermal Stress Using Weather and Physiological Variables. Computers and Electronics in Agriculture, 127, 176-183. doi:10.1016/j.compag.2016.06.014

Sousa, R. V., Rodrigues, A. V. S., Abreu, M. G., Tabile, R. A., \& Martello, L. S. (2018). Predictive Model Based on Artificial Neural Network for Assessing Beef Cattle Thermal Stress Using Weather and Physiological Variables. Computers and Electronics in Agriculture, 144, 37-43. doi:10.1016/j.compag.2017.11.033

Strutzke, S., Fiske, D., Hoffmann, G., Ammon, C., Heuwieser, W., \& Amon, T. (2019). Development of a Noninvasive Respiration Rate Sensor for Cattle. Journal of Dairy Science, 102(1), 690-695. doi:10.3168/ jds.2018-14999 PMID:30415860

Talavera, J. M., Tobón, L. E., Gómez, J. A., Culman, M. A., Aranda, J. M., Parra, D. T., \& Garreta, L. E. et al. (2017). Review of IoT Applications in Agro-Industrial and Environmental Fields. Computers and Electronics in Agriculture, 142, 283-297. doi:10.1016/j.compag.2017.09.015

USDA. National Agricultural Statistics Service, (2015). Death Loss in U.S Cattle and Calves due to Predator and Nonpredator Causes. United States Department of Agriculture.

Widmar, D. (2018). Where's the Meat? U.S. Meat Consumption Continues Higher. AG Economics. Retrieved from http://ageconomics.com/2018/02/19/wheres-meat-u-s-meat-consumption-continues-higher 
Ricardo Ribeiro Santos is with the College of Computing of the Federal University of Mato Grosso do Sul. His research interests include: IoT, digital circuits, design and automation systems, computer architecture.

Ricardo Aguiar is the co-founder and computer engineer at Indext, a startup focused on software and hardware solutions for agriculture and livestock. Master student of Computational Technologies for Agriculture and Livestock at Federal University of Mato Grosso do Sul.

Wellington Oliveira Santos is a computer engineer at Fundação Universidade Federal de Mato Grosso do Sul, cofounder of the Brazilian startup Indext, and developer at Tecsinapse. Finalist of Brazilian Intel Embedded Systems Competition 2015 - SBESC, won the award of third place in the Latin American Robotics Competition (LARC) of 2012. He also has experience in algorithms, teaching mathematics logic in Pró-Nível project, and design of CPUs in multi-level logic.

Rafael Alves Costa is a computer engineering from the Federal University of Mato Grosso do Sul. 